\title{
CARACTERIZACIÓN SOCIO-ECONÓMICA Y AMBIENTAL EN PEQUENOS Y MEDIANOS PREDIOS GANADEROS EN LA REGIÓN DEL SUMAPAZ, COLOMBIA
}

\author{
CHARACTERIZATION OF SOCIO-ECONOMIC \\ AND ENVIRONMENTAL FEATURES IN SMALL AND \\ MEDIUM LIVESTOCK FARMERS IN THE COLOMBIAN \\ SUMAPAZ REGION
}

\begin{abstract}
Charles E. Bermúdez ${ }^{1}$, Nelson E. Arenas ${ }^{2}$, Vilma Moreno Melo ${ }^{3}$
${ }^{1}$ Lic. en Educación Básica con énfasis en Ciencias Sociales, cM.Sc. Geografía, Asesor, Dirección de Investigación. Universidad de Cundinamarca, Dg 18 No. 20-29, Fusagasugá-Cundinamarca, Colombia, e-mail: cebermudezg@unal.edu.co; ${ }^{2}$ Lic. en Biología y Educación Ambiental, Ph.D. en Bioquímica y Biología Molecular, Docente programa de Zootecnia, Facultad de Ciencias Agropecuarias. Universidad de Cundinamarca, Dg 18 No. 20-29, Fusagasugá-Cundinamarca, Colombia, e-mail: nearenass@unal.edu.co; ${ }^{3}$ Zootecnista, Ph.D. en Biotecnología, Decana, Facultad de Ciencias Agropecuarias. Universidad de Cundinamarca, Dg 18 No. 20-29, Fusagasugá-Cundinamarca, Colombia, e-mail: vilma@mail.unicundi.edu.co
\end{abstract}

Rev. U.D.C.A Act. \& Div. Cient. 20(1): 199-208, Enero-Junio, 2017

\section{RESUMEN}

Las comunidades ganaderas, a pequeña y mediana escala, en la provincia del Sumapaz, se encuentran en un periodo de transición hacia la tecnificación en la producción. Así, el objetivo de este estudio fue describir algunos aspectos que actúan como barreras en la tecnificación y limitan la implementación de buenas prácticas ganaderas. Se realizó un diagnóstico participativo en pequeños y medianos ganaderos del Sumapaz, mediante encuesta aleatoria, en nueve municipios de la provincia. Los resultados evidencian que existen diferentes barreras socio-económicas, que obstaculizan el acceso a tecnologías para el mejoramiento de la producción, como el bajo nivel de ingresos económicos, acceso a la educación superior y falta de registros contables y de sanidad en la producción; sin embargo, se observaron indicadores positivos, asociados al manejo ambiental de los recursos naturales, como parte de su legado cultural.

Palabras clave: Desarrollo de la comunidad, trabajadores rurales, economía rural, aislamiento social.

\section{SUMMARY}

Livestock community from the Sumapaz region are in a transition period towards to technical production. Thus, our aim was to assess the mean requirements which delay implementation of good farming practices. This study, involved small and medium livestock farmers from the Sumapaz region through a random test applied in nine municipalities from the province. Results showed socioeconomic barriers which limited the technology transfer for improvement in production such as low income, restricted access to university education and lack of productive and sanitary records. However, we observed positive outcomes with regard to environmental management which is characteristic as their cultural heritage.

Key words: Community development, rural workers, rural economy, social isolation.

\section{INTRODUCCIÓN}

Las pequeñas comunidades rurales de la región de Sumapaz se han dedicado, tradicionalmente, a la agricultura y a la ganadería, que constituye la economía campesina, a pequeña escala, altamente vinculada al mercado de alimentos de la región, como la principal forma de producción. Estas unidades de producción son, al mismo tiempo, nichos de consumo, ya que buscan garantizar el autoabastecimiento y la reproducción social de la familia (Álvarez, 2003; Giraldo, 2008; Santacoloma-Varón, 2015).

Como proceso de adaptación e inserción del campesinado al modo de producción capitalista, las fincas ganaderas se han visto en la necesidad de mejorar sus sistemas productivos, para la obtención de carne y de leche, a través de la tecnificación del proceso, en busca de mayor eficiencia en 
la producción, rentabilidad económica y como respuesta a los mecanismos de control sanitario e inocuidad, que establecen las entidades reguladoras de inocuidad en alimentos (Fafchamps et al. 1998; Ogunmefun \& Achike, 2015). Lo anterior, exige reconvertir la ganadería en un sistema más competitivo, lo cual, implica no solo la adopción de la reglamentación impuesta por los entes estatales reguladores, sino también, un cambio en la forma de producción, respecto a manejo de suelos, pasturas, cuidado ambiental, bienestar animal y, sobre todo, lo referente al estatus sanitario y productivo de los animales (Lapar et al. 2003; Godfray \& Garnett, 2014).

Se reconoce que Colombia ha presentado una ganaderización del sector rural, entendido como una expansión permanente de la ganadería bovina en la frontera agropecuaria y un uso extensivo del suelo, en esta actividad (Betancur et al. 2016; Piniero, 2016). Para el 2011, en el departamento de Cundinamarca, el porcentaje de cabezas de ganado fue de $5,17 \%$ respecto al total nacional, con un inventario bovino de 1.267 .554 cabezas, para los 116 municipios. Dicha cifra porcentual ha permanecido relativamente constante en los últimos años, con una población bovina registrada de 1.256.535 animales (PNUD, 2011; ICA, 2016).

En términos socio-económicos, los pequeños productores de las unidades de producción ganadera (UPG) de la región del Sumapaz buscan suplir sus necesidades básicas y producir excedentes para la comercialización y la consolidación de mercados locales (Santacoloma-Varón, 2015). El sector es muy heterogéneo, con diferencias marcadas en el nivel de vida de los ganaderos y competitividad en el mercado. En la actualidad, se reconoce la importancia de promover el vínculo entre los pequeños y medianos productores de las UPG con el mercado nacional y global, como estrategia encaminada al desarrollo y al incremento de ingresos, ante el alto índice de pobreza que existe entre los pobladores rurales, que alcanza una tasa de incidencia de, aproximadamente, el 49\% (PNUD, 2011).

Además, se debe tener en cuenta la ventaja competitiva que puede ofrecer este sector en la producción, con demanda creciente, que representa una estrategia para estimular un mejor manejo de los recursos naturales, por parte del productor rural (Ostertag-Gálvez, 1999). Con base en dicho contexto, se describen algunas características socio-económicas de los pequeños y medianos productores ganaderos del Sumapaz, que contribuyen a conocer la dinámica de desarrollo de esta población rural. Así, es importante conocer la eficiencia en los sistemas de producción pecuaria, para diseñar estrategias, encaminadas a aumentar los beneficios económicos y la rentabilidad. Aunque, si bien el Estado intenta promover la transición del productor campesino ha- cia el pequeño empresario, no es consecuente en la política social y el modelo económico que tienden a marginalizarlo como grupo social (Rodríguez- Espinosa et al. 2016).

El propósito de este estudio, se centró en la reflexión sobre las características socio-económicas de pequeños y medianos productores, quienes podrían representar barreras para la transferencia de tecnologías y capacitación en buenas prácticas ganaderas (BPG). Teniendo en cuenta que es necesario ser eficiente y generar producción bovina de calidad e inocuidad para poder acceder a los exigentes mercados nacionales e internacionales, se hace necesaria la comprensión y la aplicación del concepto integral de salud animal, prevención, bienestar y manejo animal, que se estructuran en el marco de las BPG (Uribe et al. 2011). Conocer técnicamente las BPG y descubrir la importancia de implementarlas en las UPG de la región constituye, en la actualidad, una gran oportunidad, ya que permite continuar una transformación importante y decidida en los sistemas de producción pecuario, con la aplicación de criterios logísticos y administrativos, que permitan un uso racional y eficiente de los recursos físicos, humanos y económicos existentes.

\section{MATERIALES Y MÉTODOS}

Zona de estudio. La provincia del Sumapaz tiene múltiples extensiones, según la perspectiva geográfica que la defina: páramo, cuenca, provincia, región y localidad son algunas de sus denominaciones. Para este estudio, se eligió como unidad de observación la provincia del Sumapaz, ubicada al sur del departamento Cundinamarca, conformada por diez municipios: Granada, Silvania, Tibacuy, Fusagasugá, Pasca, Arbeláez, San Bernardo, Pandi, Venecia y Cabrera. Todos los municipios forman parte de la cuenca hidrográfica del río Sumapaz, que nace en el Páramo del mismo nombre. La provincia tiene una extensión territorial de $1.808 \mathrm{~km}^{2}$, representando el $8 \%$ del área total de Cundinamarca, lo que le permite posicionarse como la sexta, en cuanto a tamaño, a nivel departamental.

En los últimos años, se reconoce un carácter multifuncional del territorio del Sumapaz cundinamarqués, haciendo referencia a las múltiples actividades económicas generadas, a partir de una mayor interrelación urbano/regional y campo/ ciudad. Esta interrelación, se manifiesta en la proliferación de actividades comerciales y de servicios, acompañada de una mayor urbanización del campo, ampliación de la oferta turística y de bienes ambientales. Otra característica asociada a la multifuncionalidad del territorio es la pluriactividad de las familias campesinas, entendida como la combinación de actividades ocupacionales, como estrategia de subsistencia, ante las condiciones precarias que caracteriza la producción agropecuaria en Colombia (Piñeros, 2014). 
Para el área de desarrollo de la provincia del Sumapaz, Bogotá es el centro económico de mayor importancia, debido al fácil acceso que tienen los municipios con la capital del país. En la región del Sumapaz predominan las tierras de pastizales, que ocupan una extensión 52.523 ha, que cubren el $28,6 \%$ de la provincia. De igual forma, se destacan extensiones de bosque secundario, que ocupan el 21,5\%; vegetación de páramo, el 14,8\%; rastrojo, el 8,3\%; pasto con rastrojo, el $5,3 \%$ y bosque natural, el 4,4\%. En el Sumapaz, tan sólo el 9,3\% de sus suelos está destinado a la agricultura (Giraldo, 2008).

Caracterización socio-económica y ambiental en pequeños y medianos predios ganaderos. Se realizó, a través de diagnóstico participativo entre 2014 y 2015, tomando como muestra 29 UPG, incluyendo 19 UPG pequeñas (con menos de 10 cabezas de ganado) y 10 UPG medianas (entre 11 y hasta 50 cabezas de ganado), localizadas en los municipios de Granada, Silvania, Tibacuy, Fusagasugá, Pasca, Arbeláez, San Bernardo, Pandi, Venecia y Cabrera. Se analizó la estructura de estas unidades, utilizando un sistema de indicadores de sostenibilidad, aplicado a espacios rurales colombianos, con énfasis en los componentes social, económico y ambiental, conforme a estudios previos (Winograd, 1995; Fawaz-Yissi \& Vallejos-Cartes, 2011). De este muestreo, se obtuvieron los siguientes indicadores: servicios básicos, como agua potable y electricidad; nivel educativo; nivel de ingresos de los hogares; uso de registros contables; tenencia de la tierra y manejo del medio ambiente.

El estudio, se ejecutó en dos fases. Inicialmente, para la obtención de la información, se realizaron visitas de campo a las UPG incluidas, a través de una encuesta semi-estructurada, observación directa, perfil de actividades cotidianas, registro de actividades y recolección de datos sociodemográficos. Posteriormente, se diseñó y se desarrolló un taller de retroalimentación con los productores, en el cual, se socializó la información recolectada y analizada. También, se elaboró un plan de trabajo para cada sistema de producción, basado en la información recolectada, que fue almacenada en una base de datos, en formato Excel (Microsoft Office), para su posterior análisis. Los resultados, se describieron y se presentaron en figuras y frecuencias numéricas, en promedios y porcentajes.

\section{RESULTADOS Y DISCUSIÓN}

Caracterización de los predios. Las 29 UPG muestreadas, se caracterizaron por la homogeneidad en los parámetros ambientales, socio-económicos y culturales, sin representar, globalmente, la comunidad rural, de su respectivo origen geográfico. El $17 \%$ de los predios, se encontraban habitados por campesinos, sin ningún vínculo familiar y en las UPG, ad- ministradas a nivel familiar. El 42,2\% de las UPG, se encontraban constituidas por cuatro integrantes como núcleo familiar, seguido por $20,5 \%$, compuesta por cinco miembros; el $12 \%$, por 3 integrantes y hasta siete integrantes, en el resto de las UPG. A pesar del abandono técnico de las instituciones gubernamentales para el desarrollo rural, el $62 \%$ de las UPG estaban inscritas en el ICA.

En la primera fase, la observación más común en todos los predios fueron las prácticas empíricas en el manejo animal y ambiental, incluyendo quemas de residuos sólidos, vertimientos puntuales a fuentes hídricas, deforestación para la extensión de la frontera agrícola, degradación de suelos y, en consecuencia, una baja viabilidad para la fauna y flora. Asimismo, se identificaron debilidades en el manejo administrativo de sus predios, debido a una deficiente autogestión y carencia de conocimientos de saneamiento básico. También, en todas las UPG incluidas, predominó la producción avícola y piscícola, junto con las actividades ganaderas, conforme a estudios previos (Giraldo, 2008). Así, las UPG abarcadas, se clasificaron de acuerdo al tipo de producción: 15 fincas, con propósito lechero y 14, doble propósito. Los productores presentaron baja capacidad de inversión económica en infraestructura y mano de obra para las UPG, dificultades para integrarse a las cadenas de mercantilización y escasa agremiación campesina. También, desconocimiento de BPG y percepciones empíricas, respecto a las prácticas pecuarias.

Servicios básicos en las fincas. En los territorios rurales latinoamericanos, el acceso a los servicios públicos puede ser limitado, por la infraestructura en los sistemas de distribución y la competencia por el acceso al recurso natural, en fuentes naturales (Cáceres \& Rodríguez-Bilella, 2014). Respecto a los servicios básicos, se observó que en las UPG incluidas, un $72,4 \%$ cuenta con servicio de luz (electricidad) y agua potable (Figura 1). Estos resultados indican que la disponibilidad de los servicios públicos continúa siendo una brecha para las comunidades rurales, la cual, se estima en un $17 \%$, para energía eléctrica y en un $45 \%$, para agua, conforme a la encuesta de calidad de vida en Colombia, de 2003 y estudios posteriores (Forero-Álvarez \& Ezpeleta-Merchán, 2007; Rodríguez-Espinosa et al. 2016; Ramírez et al. 2016). Además, se observó la implementación de pozo séptico $(93,1 \%)$ y la disponibilidad del servicio de baño, en la mayoría de los predios (96,5\%) (Figura 1). En otros contextos rurales, similares a Colombia, la carencia de servicios públicos adecuados, se podría convertir en un factor que promueve la migración hacia centros urbanos, por el deterioro de las condiciones de reproducción social campesinas (Cáceres \& Rodríguez-Bilella, 2014; Kale \& Attar, 2016; Piniero, 2016). Asimismo, la disponibilidad de servicios básicos, como luz y agua potable, podrían ser un factor limitante en la implementación de BPG o en la tecnificación de la producción de los predios. 


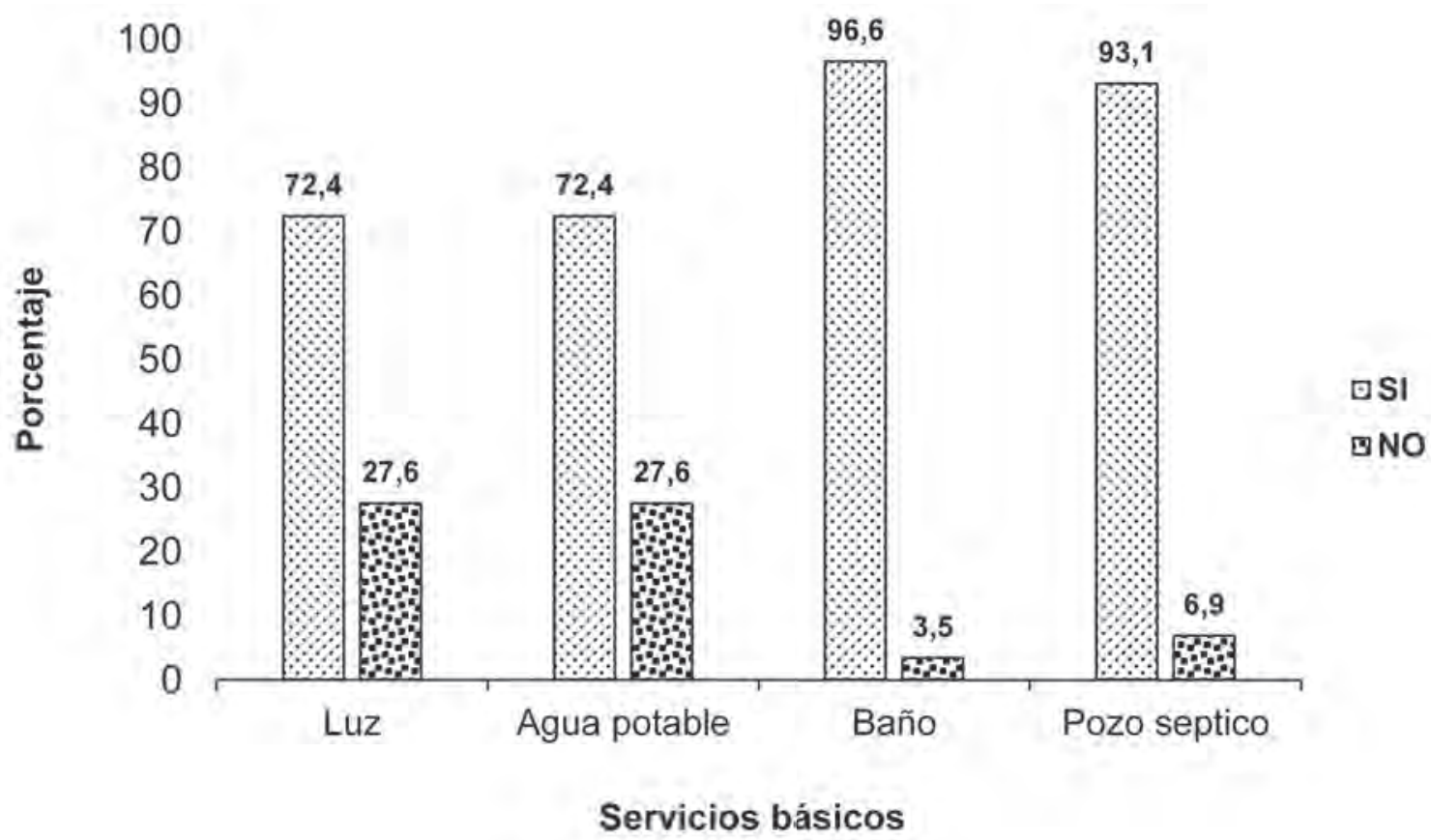

Figura 1. Disponibilidad de servicios básicos en UPG rurales del Sumapaz, incluidas en este estudio.

Nivel educativo de las comunidades campesinas. Para el análisis de este campo, se tomaron como indicadores: el acceso a la educación y el nivel educativo de productores, con lo cual, se describió la estructura educativa para las familias. Del total de familias encuestadas, el 96,5\% señaló que tienen acceso al sistema educativo de educación primaria, básica secundaria y media, es decir, todos los municipios cuentan con infraestructura para atender a la población, en edad escolarizada. Si bien se destaca que la extensión de matrícula en educación ha sido continua, se evidencia la existencia de barreras socio-económicas, que limitan el acceso a la educación superior y que persisten hacia la desigualdad campo/ ciudad, en temas de calidad y de cobertura; la segmentación escolar publico/privada, así como restricciones a nivel de género, respecto al acceso y a la moratoria educativa (FawazYissi \& Vallejos-Cartes, 2011; Ramírez et al. 2016).

Es así, que se marca una diferenciación en el nivel educativo entre propietarios y encargados o responsables de las fincas. Para el caso de los primeros, predomina un nivel educativo profesional, con un $44,8 \%$ (Figura 2a), mientras que para los segundos, el nivel educativo que sobresale es la educación básica primaria, con un $41 \%$ (Figura 2b). Esta desigualdad, se podría relacionar con la capacidad de movilidad, de tiempo y de recursos económicos con los que disponen los productores para acceder a la educación superior, así como a la oferta educativa, que se centra, en su mayoría, en los cen- tros urbanos, lo que constituye una barrera socio-espacial (Schady et al. 2015). La existencia de estas barreras limita la implementación, por parte de productores, de modelos más eficientes y planificados en el desarrollo de sus actividades diarias, ya que muchos productores carecen de la formación técnica y profesional para implementar tecnologías en sus predios, para llevar registros contables y evaluaciones periódicas y tener un manejo sanitario óptimo de sus prácticas y productos (López-Arellano \& Montoya-Grajales, 2016).

Nivel de ingresos, tenencia de la tierra y registros contables. Típicamente, las familias campesinas se adaptan a las formas de reproducción social y económica, establecidas por las condiciones estructurales del mercado y el modelo económico (Llambí \& Pérez, 2007). Las actividades agropecuarias de los pequeños y medianos productores incluidos, las realizan en parcelas, con un promedio de 5 ha, como extensión típica del minifundio. Allí, se desarrollan diferentes actividades socio-económicas, que definen una economía familiar, caracterizada por la pluriactividad, donde, además de los ingresos propios de la explotación agropecuaria, se obtienen otros, denominados extraprediales, como la venta de mano de obra fuera de la finca, el arriendo de los predios, servicios turísticos, empleos rurales no agrícolas y empleos tercerizados (Méndez-Sastoque, 2015). Esta realidad contrasta con la imagen típica que se tiene del campesino confinado en los espacios rurales, pues no solo son evidentes 
a

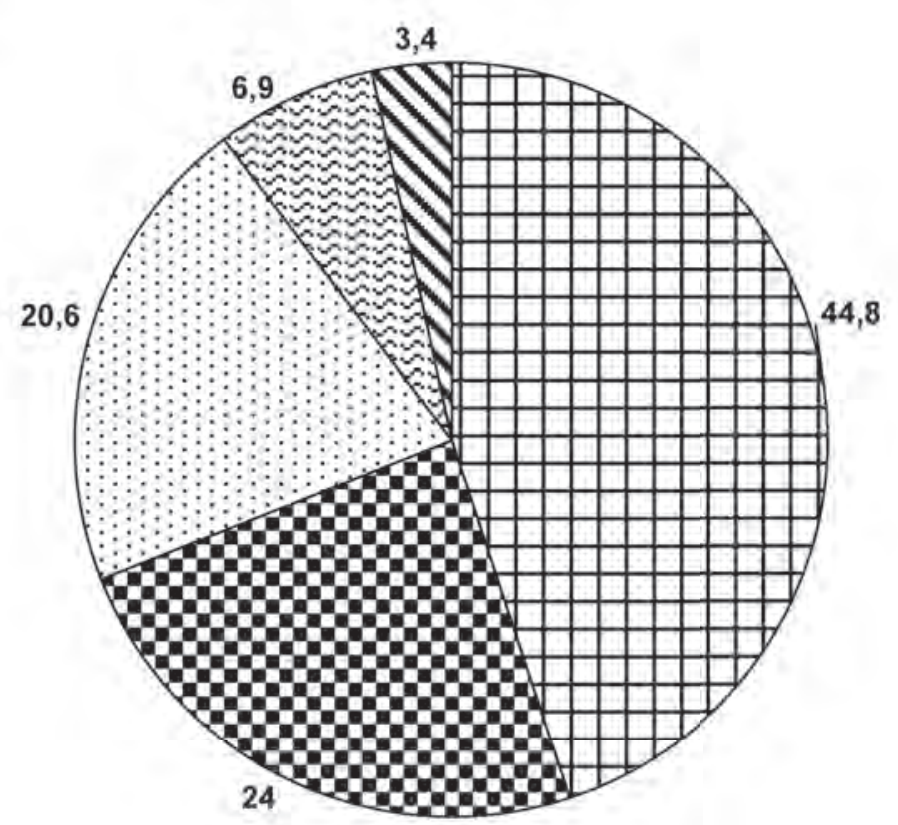

$\square$ Profesional

QBachiller

¿Básica primaria

日Técnico

$\triangle$ Sin estudio

b

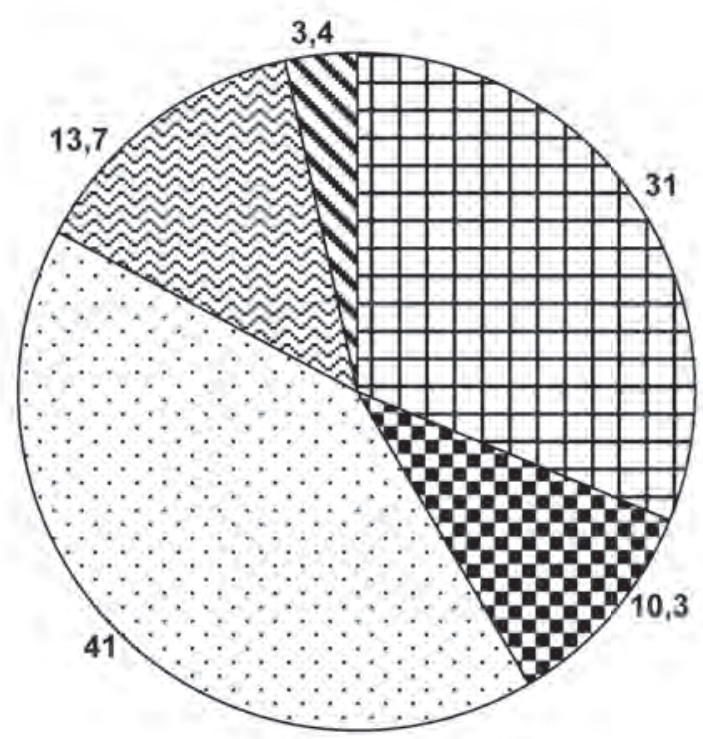

๓ Profesional

Bachiller

口Básica primaria

घTécnico

घ Sin estudio

Figura 2. a. Nivel educativo de los propietarios de las fincas (expresados en porcentajes) y b. Nivel educativo de los arrendatarios de las fincas.

las transformaciones productivas de las economías campesinas, sino que también sobresalen los cambios socio-culturales, producto de un mayor intercambio cultural y mercantil con la economía de mercado y las ciudades (Forero-Álvarez, 2002).
En cuanto al nivel de ingresos económicos, se tiene que el $55,2 \%$ de los productores encuestados obtienen ingresos mensuales entre 1 y 2 salarios mínimos legales vigentes (SMLV); para el $24,1 \%$, su nivel de ingresos mensuales es de menos de 1 SMLV y solo para el $20,7 \%$, está entre 3 y 4 SMLV (Figura 3). El mayor ingreso económico proviene de la 
venta de leche, con el 67\%; asimismo, el $61 \%$ de los productores realizan la comercialización de ganado y de leche en la finca y mercado local. Además, se identificó que aún no existe la organización empresarial o a nivel de cooperativas y que no han implementado ninguna tecnología para mejorar los procesos de comercialización. Respecto a la posesión de predios, se registró en la zona que el $90 \%$ de los productores ganaderos son dueños de la tierra y solo un $10 \%$ son arrendatarios. En estudios previos en la región del Sumapaz, se reportó una tendencia similar respecto a la propiedad de los predios (Giraldo, 2008), sin embargo, los análisis respecto al uso y tenencia de la tierra demuestran que no solo la propiedad sobre los predios aumenta las posibilidades de inserción al mercado y al desarrollo productivo, sino que también lo son el poder acceder al capital, a créditos y a servicios básicos de calidad, los cuales, se convierten en elementos imprescindibles para aliviar la pobreza rural y promover la tecnificación de la producción (PNUD, 2011).

En cuanto al manejo de registros productivos, se evidenció que los productores no llevan controles periódicos sobre las actividades pecuarias y existe un desconocimiento generalizado de prácticas contables. Aunque el $58,6 \%$ de los predios encuestados sí implementan algún tipo de registro de producción, tan solo el $22,1 \%$ efectúa registro de gastos y el $27,6 \%$ de ventas, con lo cual, es difícil calcular los costos totales de inversión y el rendimiento de utilidades de la producción (Figura 4). Las facilidades crediticias para la financiación de la producción no son factibles para los pequeños productores y, al contrario, pueden afectar a la propiedad raíz. Dichas limitaciones han sido descritas en productores agropecuarios, en otros contextos socio-económicos (Khattak \& Hussain, 2008; Rivera \& Monroy, 2015).

Ambiente y manejo de recursos naturales. La herramienta de diagnóstico utilizada fue una lista de chequeo, que evaluó, porcentualmente, la calidad de las prácticas desarrolladas en el marco del impacto ambiental de la actividad productiva. Para evaluar el nivel de desarrollo de las UPG, se elaboró una escala (Tabla 1), que mide el impacto ambiental y actividades armoniosas con el ambiente, aplicada al contexto rural colombiano (Winograd, 1995; Fawaz-Yissi \& Vallejos-Cartes, 2011). El diagnóstico permitió establecer que un $63,8 \%$ de las UPG realiza prácticas adecuadas en relación con la sostenibilidad ambiental; se destaca la implementación de cercas vivas -como corredores ecológicos-, tratamiento de aguas residuales domésticas, creación de sistemas de bioabonos o compostaje, manejo integral de residuos sólidos y peligrosos, rotación de pradera continua y protección de fauna y flora. Las prácticas negativas, en un $36,2 \%$, se relacionaron con quemas de residuos sólidos, vertimientos domésticos, deforestación para potreros, contaminación de rondas hídricas, compactaciones de suelo y lodazales. Algunas de las mencionadas prácticas contribuyen a la extensión de la frontera agrícola o ganadera e intensificación de esta actividad económica (Piniero, 2016). Dichas prácticas han sido descritas en otras comunidades rurales de diferentes áreas geográficas y contextos, asociados con menor disponibilidad de recursos ecosistémicos (Hartel et al. 2014; Goulart et al. 2016).

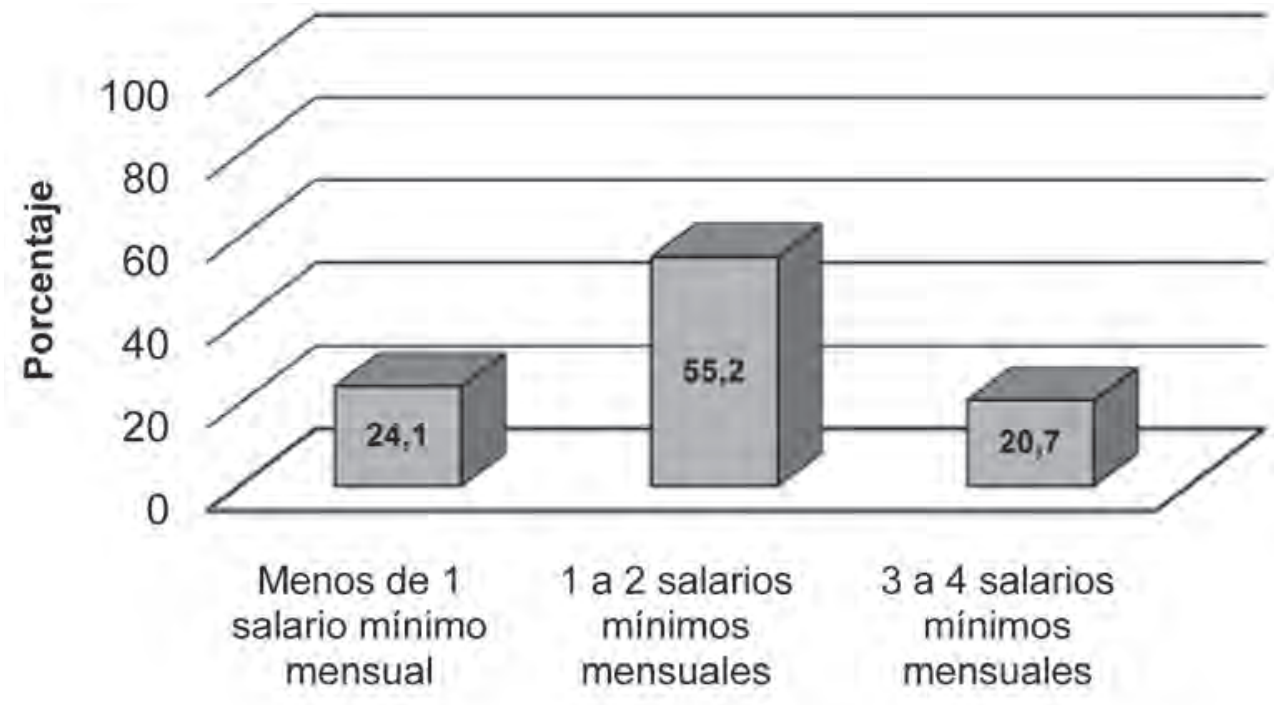

Ingreso salarial

Figura 3. Nivel de ingresos de pequeños y medianos productores campesinos de 29 UPG, en la región del Sumapaz. 


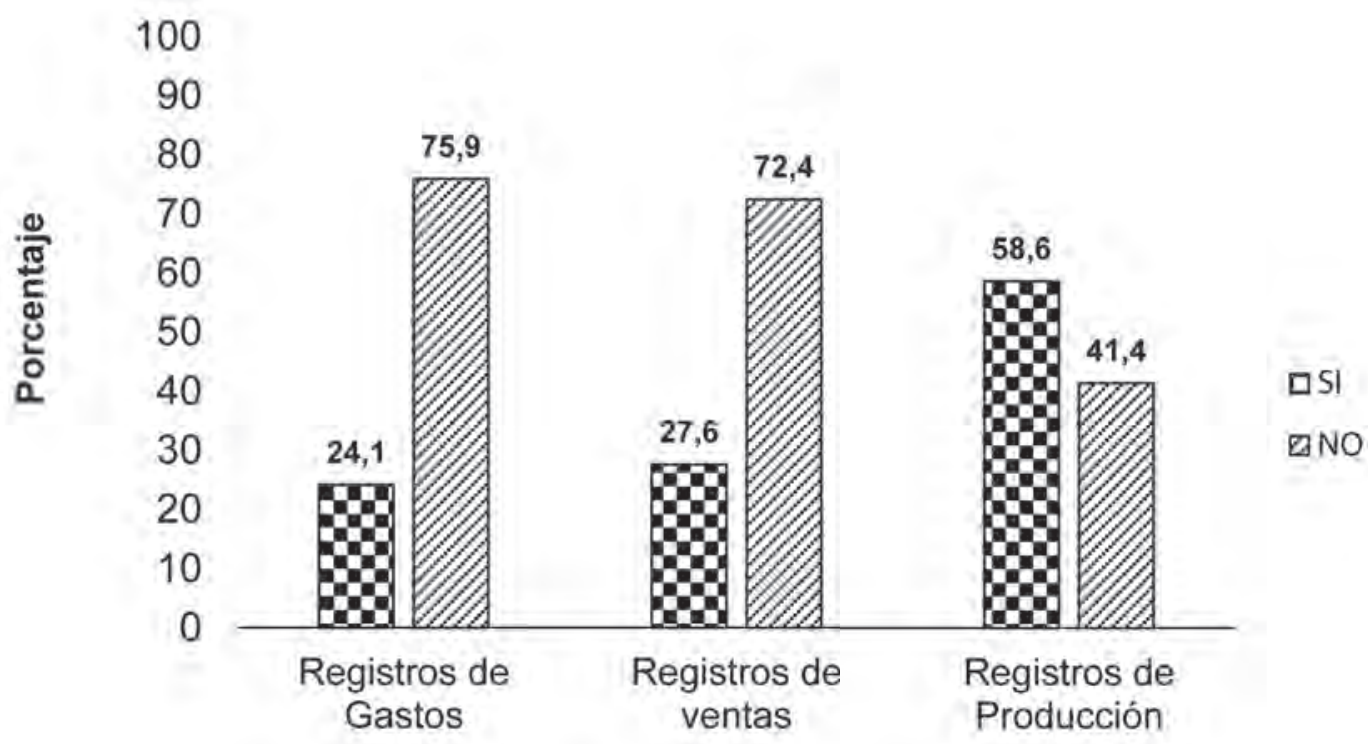

Tipo de registro

Figura 4. Implementación de registros contables por parte de productores campesinos del Sumapaz.

Tabla 1. Escala de calificación ambiental. Calificación en vía del desarrollo sostenible.

\begin{tabular}{|l|c|c|}
\hline \multicolumn{1}{|c|}{ Calificación } & $\begin{array}{c}\text { Procentaje de calificación } \\
\text { en la matriz }\end{array}$ & $\begin{array}{c}\text { Parámetro +/ 10\% de mejora } \\
\text { continua ambiental }\end{array}$ \\
\hline Excelencia ambiental (sostenible) & $86-99$ & $-10 \%$ \\
\hline Consistente ambiental & $66-85$ & $-10 \%$ \\
\hline Media ambiental & $46-65$ & 0 \\
\hline Sobresaliente ambientalmente & $26-45$ & $10 \%$ \\
\hline Deficiencia ambiental & $5-25$ & $10 \%$ \\
\hline
\end{tabular}

En cuanto al manejo de los suelos, solo en el $20,7 \%$ de las UPG han realizado, recientemente, análisis del suelo y únicamente el 13,8\% registraron análisis de agua. Este indicador sugiere un desconocimiento generalizado de los productores frente al uso de técnicas, para asegurar la inocuidad en la producción y evitar plagas y enfermedades, causadas por patógenos ambientales. La anterior explicación concuerda, a su vez con el manejo integrado de plagas, que solamente es realizado en el $17,2 \%$ de las UPG. El conocimiento del ambiente y optimización de los recursos naturales puede ser un parámetro clave para aumentar la producción, especialmente, aquella basada en monocultivo y disminuir el impacto antrópico sobre el ecosistema (Villa-Méndez et al. 2008).
La transferencia efectiva de tecnologías y de capacitación en buenas practicas ganaderas exige que las unidades de producción cumplan con condiciones sanitarias mínimas, así como la disponibilidad de recursos y de equipos, para acondicionar las fincas en unidades económicas más rentables (Ayalew et al. 2013). Como se ha visto, existen limitaciones financieras, como el bajo nivel de ingresos de los productores que podrían explicar la escasa inversión en asistencia técnica y el predomino de prácticas pecuarias tradicionales (Schady et al. 2015). Este escenario plantea la necesidad de una mayor intervención y apoyo técnico y social de las instituciones públicas, pues hasta ahora, la preocupación solo ha sido tecnificar los procesos sin considerar las condiciones sociales, propias de la población rural (López-Arellano 
\& Montoya-Grajales, 2016). Esta situación va acompañada de un alto nivel de descapitalización entre los productores, quienes, también, se muestran indiferentes o temerosos a la utilización del crédito bancario. Además, las limitaciones respecto a los niveles de producción y deficiencias en la respectiva calidad, podrían explicar su escaso poder de negociación en el mercado, como se ha descrito en comunidades rurales, con contextos socio-culturales similares (Somda et al. 2005).

Es necesario destacar que una de las características de las familias rurales del Sumapaz es su arraigo con la economía campesina (Giraldo, 2008). Esto supone, que si bien es necesario la implementación de sistemas productivos que garanticen una mayor inserción y competencia en el mercado, ésta no debe desconocer características, como la soberanía alimentaria, el vínculo con la tierra y la ética del trabajo campesino, aspectos que reafirman la identidad tradicional de este grupo social y que se constituyen como ventajas ante las formas de invisibilización y marginación, por parte de la institucionalidad (Santacoloma-Varón, 2015). Son importantes los indicadores de acceso a servicios públicos y educación, pues se consideran factores determinantes al momento de evaluar la calidad de vida de los productores y sus familias. Asimismo, es sobresaliente el indicador de buenas prácticas ambientales, acorde con la tradición campesina de preservación y buen manejo de los recursos naturales.

La implementación de BPG en la provincia de Sumapaz, se constituye en una labor que beneficia a toda la comunidad participante en las UPG; para su adopción o implementación, se requirió, primero, estudiar las características socio-demográficas de la población y caracterizar los predios, para realizar una intervención efectiva, en términos de transferencia tecnológica y de capacitación, en la segunda fase del estudio. Los fundamentos sobre los cuales se logró implementar exitosamente las BPG en 24 UPG (82,7\%), se basaron en la importancia del manejo del suelo vivo y su balance, pastoreo, nutrición animal, agua en los sistemas, manejo genético, sanidad/medicación, equipos adecuados, correcta interacción con el medio ecológico y social, además de agregar valor y optimizar las cadenas de comercialización (Grote, 2014; Godfray \& Garnett, 2014). Es posible que los resultados observados en este estudio apliquen no solo a las prácticas ganaderas, sino también adapten a otras prácticas pecuarias, como la porcícola, avícola y piscícola, que se desarrollan en el Sumapaz, por pequeños y medianos productores. Igualmente, el desarrollo socio-económico de las comunidades del Sumapaz se podría potenciar, a través de las alianzas con otros sectores, como academia, empresas privadas y sector productivo.

Agradecimientos: Los autores expresan sus agradecimientos a Diego Abril y Paola Valencia, por su colaboración en la recolección de la información. También, a toda la comunidad rural de las fincas que participaron en el estudio. Conflictos de intereses: El manuscrito fue preparado y revisado con la participación de todos los autores, quienes declaramos que no existe conflicto de intereses que ponga en riesgo la validez de los resultados presentados. Financiación: Este estudio fue financiado por el Comité de ganaderos de la provincia del Sumapaz (COMIGAN), la Asociación Colombiana para el Avance de la Ciencia (ACAC) y la Universidad de Cundinamarca, en el marco de la segunda fase del proyecto de transferencia efectiva de tecnologías a pequeños productores agropecuarios en la provincia de Sumapaz (Cundinamarca, Colombia).

\section{BIBLIOGRAFÍA}

1. ÁLVAREZ, J.F. 2003. Economía campesina y sistema alimentario en Colombia: Aportes para la discusión sobre seguridad alimentaria. Facultad de Estudios Ambientales y Rurales. Ed. Universidad Javeriana (Bogotá, Colombia). 44p.

2. AYALEW, T.; DUGUMA, B.; TOLEMARIAM, T. 2013. Socioecomic and farm characteristics of smallholder cattle producers in Ilu Aba zone of Oromia regional state, South Western Ethiopia. Global Vet. (Dubai). 10(5):607-613.

3. BETANCUR, F.R.; ESPINOSA, H.R.; SIERRA, M.M. 2016. Dinámica de la superficie agrícola cultivada en Colombia, 1960-2010. UGCiencia. (Colombia). 22(1):85-98.

4. CÁCERES, D.M.; RODRÍGUEZ-BILELLA, P. 2014. Acceso y apropiación del agua en comunidades rurales pobres de Argentina central: Transformaciones y conflictos. EST. (Mexico). 14(45):359-395.

5. FAFCHAMPS, M.; UDRY, C.; CZUKAS, K. 1998. Drought and saving in West Africa: are livestock a buffer stock?. J. Dev. Econ. (Netherlands). 55(2):273-305.

6. FAWAZ-YISSI, J.; VALLEJOS-CARTES, R. 2011. Calidad de vida, ocupación, participación y roles de género: un sistema de indicadores sociales de sostenibilidad rural (Chile). Cuad. Desarro. Rural. (Colombia). 8(67):45-68.

7. FORERO-ÁLVAREZ, J. 2002. La economía campesina colombiana 1990-2001. Cuadernos tierra y justicia No. 2. Instituto Latinoamericano para una Sociedad y un Derecho Alternativos-ILSA (Bogotá, Colombia). 32p. 
8. FORERO- ÁLVAREZ. J.; EZPELETA-MERCHÁN, S. 2007. Las brechas entre el campo y la ciudad en Colombia 1990-2003, y propuestas para reducirlas. Ed. Naciones Unidas, CEPAL, serie Estudios y Perspectivas 17. (New York, USA). 79p.

9. GIRALDO, O.F. 2008. Seguridad alimentaria y producción pecuaria campesina: el caso de la localidad rural de Sumapaz. Luna Azul. (Colombia) 27:49-59.

10. GODFRAY, H.C.J.; GARNETT, T. 2014. Food security and sustainable intensification. Phil. Trans. R. Soc. B. (UK). 369:20120273.

11. GOULART, F.F.; CARVALHO-RIBEIRO, S.; SOARESFILHO, B. 2016. Farming-biodiversity segregation or integration? revisiting land sparing versus land sharing debate. J. Environ. Prot. (USA). 7:10161032.

12. GROTE, U. 2014. Can we improve global food security? A socio-economic and political perspective. Food Security. (Netherlands). 6(2):187-200.

13. HARTEL, T.; FISCHER, J.; CÂMPEANU, C.; MILCU, A.I.; HANSPACH, J.; FAZEY, I. 2014. The importance of ecosystem services for rural inhabitants in a changing cultural landscape in Romania. Ecol. Soc. (Canada). 19(2):42-51.

14. ICA. 2016. Censo Pecuario Nacional. Bogota, Colombia. Disponible desde Internet en: http://www.ica.gov.co/ getdoc/8232c0e5-be97-42bd-b07b-9cdbfb07fcac/ Censos-2008.aspx (con acceso 12/04/2016).

15. KALE, M.T.S.; ATTAR, A.C. 2016. Socio-Economic Survey of Gotkhindi Village. IJIR. (Dubai) 2(9):148151.

16. KHATTAK, N.U.R.K.; HUSSAIN, A. 2008. An analysis of socioeconomic profile of rice farmers in rural economy of District Swat. Sarhad J. Agric. (Pakistan) 24(2):377-382.

17. LAPAR, M.L.; BINH, V.T.; EHUI, S. 2003. Identifying barriers to entry to livestock input and output markets in Southeast Asia. Livestock sector report Vietnam. Livestock Information, Sector Analysis and Policy Branch (AGAL). Food and Agriculture Organization of the United Nations. Ed. FAO Asia-Pacific (Vietnam). 46p. Disponible desde Internet en: http://www.fao. org/ag/againfo/resources/en/publications/sector_ reports/lsr_VNM.pdf (con acceso 18/09/2016).
18. LLAMBÍ, I.L.; PÉREZ, C.E. 2007. Nuevas ruralidades y viejos campesinismos. Agenda para una nueva sociología rural latinoamericana. Cuad. Desarro. Rural. (Colombia). 4(59):37-61.

19. LÓPEZ-ARELLANO, H.; MONTOYA-GRAJALES, W.D. 2016. Análisis de la política de transformación productiva colombiana, basada en la incorporación de la ciencia, la tecnología y la innovación, para el mejoramiento de la competitividad de Colombia entre los años 2010 y 2014. Rev. Electr. Educare. (Costa Rica). 20(3):98-119.

20. MÉNDEZ-SASTOQUE, M.J. 2015. Ocupación rural no agrícola y desarrollo rural local: reflexiones y aportes para una articulación efectiva. Interações (Campo Grande). (Brazil). 16(2):315-325.

21. OgUNMEFUN, S.O.; ACHIKE, A.I. 2015. Profitability analysis of selecting informal insurance measures for selected enterprises by rural farmers in odogbolu local government area. RJOAS. (Rusia). 39(3):25-36.

22. OSTERTAG GÁLVEZ, C.F. 1999. Identificación y evaluación de oportunidades de mercado para pequeños productores rurales: Centro Internacional de Agricultura Tropical (CIAT). Cali, Colombia. v. (Instrumentos metodológicos para la toma de decisiones en el manejo de los recursos naturales 7). 180p. Disponible desde internet en: http://ciatlibrary.ciat.cgiar.org/Articulos_ciat/agroempresas/ instrumentos_metodologicos.pdf (con acceso 18/09/2016).

23. PINIERO, M.C. 2016. Globalization and industrialization of agriculture: impacts on rural Chocontá, Colombia. Luna Azul. 43:468-496.

24. PIÑEROS, R. 2014. Economías campesinas y juventudes en la provincia del Sumapaz Cundinamarqués: Trayectorias sociales y relatos de vida. Ed. Universidad de Cundinamarca (Fusagasuga, Cundinamarca). p.9-35.

25. PNUD. 2011. Colombia rural. Razones para la esperanza. Programa de las Naciones Unidas para el Desarrollo. Bogota, Colombia. 42p. Disponible desde Internet en: http://escuelapnud.org/biblioteca/documentos/ abiertos/06_indh2011co.pdf (con acceso 20/05/2016).

26. RAMÍREZ, J.; CARLOS, J.; PARDO, R.; ACOSTA, O.L.; URIBE, L.J. 2016. Bienes y servicios públicos 
sociales en la zona rural de Colombia: brechas y políticas públicas. ONU, CEPAL y DNP: Serie estudios y perspectivas. Bogota, Colombia. 76p. Disponible desde Intenet en: http://200.9.3.98/ handle/11362/38948 (con acceso 20/12/2016).

27. RIVERA, J.J.M.; MONROY, H.C. 2015. Diversificación de ingresos en el sector rural y su impacto en la eficiencia: evidencia para México. Cuad. Desarro. Rural. (Colombia). 12(76):57-81.

28. RODRÍGUEZ-ESPINOSA, H.; RAMÍREZ GÓMEZ, C.J.; RESTREPO-BETANCUR, L.F. 2016. Análisis comparativo de la dinámica de desarrollo agrícola en suramérica en el período 1980-2010. Luna Azul. 42:15-29.

29. SANTACOLOMA-VARÓN, L.E. 2015. Importancia de la economía campesina en los contextos contemporáneos: una mirada al caso colombiano. Entramado. (Colombia) 11(2):38-50.

30. SCHADY, N.; BEHRMAN, J.; ARAUJO, M.C.; AZUERO, R.; BERNAL, R.; BRAVO, D.; LOPEZ-BOO, F.; MACOURS, K.; MARSHALL, D.; PAXSON, C.; VAKIS, R. 2015. Wealth gradients in early childhood cognitive development in five Latin American countries. J Hum Resour. (USA) 50(2):446-463.
31. SOMDA, J.; KAMUANGA, M.; TOLLENS, E. 2005. Characteristics and economic viability of milk production in the smallholder farming systems in The Gambia. Agric. Syst. (UK). 85(1):42-58.

32. URIBE, F.; ZULUAGA, A.F.; VALENCIA, L.; MURGUEITIO, E.; OCHOA, L. 2011. Buenas prácticas ganaderas. Manual 3, Proyecto Ganadería Colombiana Sostenible. GEF, BANCO MUNDIAL, FEDEGÁN, CIPAV, FONDO ACCION, TNC. (Bogotá, Colombia). 82p. Disponible desde Internet en: http://www.cipav. org.co/pdf/3.Buenas.Practicas.Ganaderas.pdf (con acceso 01/06/2016).

33. VILLA-MÉNDEZ, C.; TENA, M.; TZINTZUIN, R.; VAL, D. 2008. Caracterización de los sistemas ganaderos en dos comunidades del municipio de Tuzantla de la región de Tierra Caliente, Michoacán. A.I.A. (Mexico). 12(2):45-58.

34. WINOGRAD, M. 1995. Indicadores ambientales para Latinoamérica y el Caribe: hacia la sustentabilidad en el uso de tierras. Proyecto IICA/GTZ, OEA, WRI. Instituto de Recursos Naturales. (San José, Costa Rica). 84p.

Recibido: Agosto 9 de 2016

Aceptado: Abril 10 de 2017

Cómo citar:

Bermúdez, Ch.E.; Arenas, N.F.; Moreno Melo, V. 2017. Caracterización socio-económica y ambiental en pequeños y medianos predios ganaderos en la región del Sumapaz. Rev. U.D.C.A Act. \& Div. Cient. 20(1): 199-208. 